\title{
Uplink Cell Capacity of Cognitive Radio Networks with Interference Outage Constraints
}

\author{
Xuemin Hong ${ }^{\dagger}$, Cheng-Xiang Wang ${ }^{\dagger}$, and John Thompson ${ }^{\dagger \dagger}$

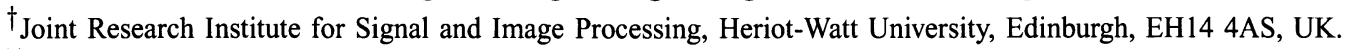 \\ ${ }^{\dagger \dagger}$ Joint Research Institute for Signal and Image Processing, University of Edinburgh, Edinburgh, EH9 3JL, UK. \\ Email: xh12@hw.ac.uk, cheng-xiang.wang@hw.ac.uk, john.thompson@ed.ac.uk
}

\begin{abstract}
Cognitive radio (secondary) networks have been proposed as a means to improve the spectrum utilization. Existing works study the capacity of cognitive radio networks as a random variable given certain peak/average interference power constraints. Such cognitive radio networks with random capacities, however, are limited in their applications since the quality of service $(\mathrm{Q} O S)$ cannot be guaranteed. In this paper we investigate the maximum stable capacity that can be provided by a cognitive radio network with interference power outage constraints. We consider a scenario where a secondary base station (BS) is located at the center while multiple secondary users are uniformly distributed within a circular cell of radius $R$. We assume that secondary users transmit on a time-division-multipleaccess (TDMA) basis and dynamically adjust their transmit power to maintain the received signal power at the BS as $P_{0}$. We first study the interference raised by such a secondary network as a random variable. Its cumulative distribution function (CDF) is derived under both simple and realistic channel models. We further assume that in order to protect primary services, the probability that the interference power perceived at a primary receiver exceeding a threshold $I_{0}$ should not be larger than an outage probability limit $\chi$. Such an interference outage constraint imposes a limit on $P_{0}$ and allows us to express the capacity as a function of $I_{0}$ and $\chi$. Moreover, the impacts of the parameters $R$, shadowing, and fading on the capacity are investigated.
\end{abstract}

\section{INTRODUCTION}

Dynamic spectrum access (DSA) has been proposed as a promising approach to improve the spectrum utilisation by allowing new wireless systems to dynamically access/share the licensed band on a negotiated or an opportunistic basis [1]. DSA strategies can be broadly categorized under three models [1]: dynamic exclusive use model, open sharing model, and hierarchical access model. In this paper, we focus on the last model which adopts a hierarchical access structure with primary and secondary users. It allows the secondary users to access the licensed spectrum under the condition that primary services are not harmfully interrupted. To achieve this, it is usually a requirement for the secondary users to be aware of the radio environment and dynamically adjust their transceiver parameters. Therefore, "secondary network" is often referred to as "cognitive radio network" in the literature [2]-[4].

A secondary network seeks to coexist with a primary network on a underlay, overlay, or interweave basis [5]. The underlay approach protects primary services by enforcing a spectral mask on the secondary signals. The interweave approach exploits the temporal and geographical dynamics of the primary signal occupation and collects the temporary or locally unused frequency voids, referred to as spectrum holes, for the use of secondary networks. The overlay approach focuses on keeping the signal-to-interference-and-noise-ratio (SINR) requirements fulfilled at primary receivers. To this end, several interference models have been proposed [6]-[10] to provide metrics of measuring the interferences raised by secondary networks. Such interferences perceived at primary receivers should fulfill certain constraints such as peak interference power constraint [11]-[13], average interference power constraint [11], [12], [14], [15], or interference outage constraint [16]. A peak interference power constraint is suitable if the interference levels at the primary receivers are known to the secondary transmitters, which can then adjust the transmit powers accordingly. In practice, secondary transmitters can obtain the information of such interference levels by means of common control channels [17] or primary receiver detection [18]. On the other hand, when the primary receivers are strictly passive and therefore "hidden" from the secondary network, it is more reasonable to consider the interference as a stochastic signal and use the average interference power constraint or interference outage constraint.

Capacity analysis is another important aspect in designing secondary networks. Unlike conventional capacity analysis for licensed networks, the capacity of a secondary network is significantly affected by the coexisting primary systems [19]. Under peak/average interference power constraints, the capacity of a secondary network is often studied as a random variable characterised by its distribution. To this end, the link and system capacity analysis of such networks are provided in [9], [11], [12] and [13]-[15], respectively. A secondary network with a random capacity, however, is restricted in its applications since the QoS of the secondary services cannot be guaranteed. It is therefore of great interest to investigate secondary networks designed to provide a stable capacity. In this paper, we will first describe a secondary network designed to provide a stable capacity by means of power control, followed by a study of the capacity of such a network under interference outage constraints.

The remainder of this paper is organized as follows. Section II describes the system model. In Sections III and IV, we study the capacity of the secondary network with simple channel models and fading channel models, respectively. Numerical results and discussions are presented in Section V. Finally, conclusions are drawn in Section VI. 


\section{SySTEM MODEL}

The system model is shown in Fig. 1 where primary users (TV receivers) and secondary users (mobile phones) coexist on a plane. We consider a secondary circular cell with a BS located at the center and multiple secondary users uniformly distributed within the cell. The cell radius is denoted as $R$. In this paper, we focus on the uplink capacity analysis of the secondary cell, while the same approach can be easily extended for downlink analysis.

We assume that multiple secondary users transmit in orthogonal channels to avoid mutual interferences. In this paper, we use the TDMA scheme, which implies that only one target secondary user is scheduled to transmit in a given time slot. It follows that such a TDMA-based secondary network is statistically equivalent to a network that has only one (target) secondary user, whose location is uniformly distributed within the cell. We refer the underlying channel from the target secondary user to the center BS as access channel. The instantaneous channel power gain from the target secondary user to the BS is denoted as $h^{A}$. Furthermore, in order to enforce a stable capacity, the target secondary user controls its transmission power $P$ so that the received signal power at the BS equals to $P_{0}$, namely,

$$
P h^{A}=P_{0} .
$$

Meanwhile, we consider an arbitrary primary receiver within the cell and denote its distance to the BS as $r(0 \leq r \leq R)$. We refer the underlying channel from the target secondary user to the primary receiver as interference channel. The instantaneous channel power gain from the target secondary user to the primary receiver is denoted as $h^{I}$. It follows that the interference perceived by the primary receiver is given as

$$
I=P h^{I}=P_{0} h^{I} / h^{A} .
$$

Clearly, $I$ should be taken as a random variable when we consider the location of the target secondary user as uniformly distributed in the cell. We further assume that in order to protect the primary services, the probability that $I$ exceeds a threshold $I_{0}$ should be smaller than an outage probability limit $\chi$. Such an interference outage constraint in turn imposes a limit on $P_{0}$ and allows us to express the capacity

$$
C=\log _{2}\left(1+P_{0} / \Omega\right)
$$

as a function of $I_{0}$ and $\chi$, where $\Omega$ denotes the total interference and noise power perceived at the BS.

\section{CAPaCity under Simple Channel Models}

Simple channel models that only consider the effect of pathloss regardless of random shadowing and fading have been adopted in some cognitive radio network studies [14], [15], [19]. The reason of using simple channel models is that they often lead to elegant analytical results which can reveal important insights without over-complicating the problem. In this section, we adopt simple channel models and aim to express $C$ given by (3) as a closed-form function of $I_{0}$ and $\chi$.
When only the pathloss is considered, we have

$$
\begin{aligned}
h^{I} & =K^{I} /\left(d^{I}\right)^{\alpha} \\
h^{A} & =K^{A} /\left(d^{A}\right)^{\alpha}
\end{aligned}
$$

where $K^{I}$ and $K^{A}$ are pathloss constants for the interference and access channels, respectively, $d^{I}$ is the distance from the primary receiver to the target secondary transmitter, $d^{A}$ is the distance from the target secondary transmitter to the BS, and $\alpha$ is the pathloss exponent. Substituting (4) and (5) into (2), it follows that

$$
I=P_{0} \frac{K^{I}}{K^{A}}\left(\frac{d^{A}}{d^{I}}\right)^{\alpha} .
$$

In (6), $P_{0}, K^{A}$, and $K^{I}$ only have scaling effects on $I$. Without loss of generality, we assume $K^{I} / K^{A}=1$. On the other hand, $d^{A}$ and $d^{I}$ are correlated random variables. Let us define a new random variable $L=\left(d^{I} / d^{A}\right)^{2}$, the CDF of $L$ can be derived as (see Appendix)

$$
F_{L}(x)=\left\{\begin{array}{lr}
\frac{k^{2} x}{(1-x)^{2}}, & x \in\left[0,(1-k)^{2}\right], \\
\frac{1}{\pi}\left[\theta_{1}+\theta_{2} \frac{k^{2} x}{(1-x)^{2}}-\frac{k \sin \theta_{1}}{|1-x|}\right], & x \in\left[(1-k)^{2}, 1\right), \\
\frac{1}{\pi} \arccos (k / 2), & x=1, \\
1-\frac{1}{\pi}\left[\theta_{1}+\theta_{2} \frac{k^{2} x}{(1-x)^{2}}-\frac{k \sin \theta_{1}}{|1-x|}\right], & x \in\left(1,(1+k)^{2}\right), \\
1-\frac{k^{2} x}{(1-x)^{2}}, & x \in\left((1+k)^{2}, \infty\right) .
\end{array}\right.
$$

where $k=r / R(0 \leq k \leq 1)$ and $\arccos (\cdot)$ is the inverse cosine function. In (7), $\theta_{1}$ and $\theta_{2}$ are given by

$$
\begin{aligned}
& \theta_{1}=\arccos \left(\frac{(1-x)^{2}+k^{2}-x k^{2}}{2 k|1-x|}\right) \\
& \theta_{2}=\arccos \left(\frac{x k^{2}+k^{2}-(1-x)^{2}}{2 k^{2} \sqrt{x}}\right)
\end{aligned}
$$

respectively. For convenience, let us rewrite (6) in the $\mathrm{dB}$ scale

$$
(I)_{d B}=\left(P_{0}\right)_{d B}-(L)_{d B}
$$

where $(I)_{d B}=10 \log _{10} I,\left(P_{0}\right)_{d B}=10 \log _{10} P_{0}$, and $(L)_{d B}=5 \alpha \log _{10} L$. By using the transformation of random variables [20], the $\mathrm{CDF}$ of $(L)_{d B}$ can be written as

$$
F_{(L)_{d B}}(x)=F_{L}\left(10^{\frac{x}{5 \alpha}}\right) .
$$

From (10) and (11), we can easily obtain the CDF of $(I)_{d B}$ as

$$
F_{(I)_{d B}}(x)=1-F_{(L)_{d B}}\left(P_{0}-x\right) .
$$

We now consider an interference outage constraint which specifies that $(I)_{d B}$ should not exceed a threshold $\left(I_{0}\right)_{d B}=$ $10 \log _{10} I_{0}$ with an outage probability of $\chi$, namely

$$
1-F_{(I)_{d B}}\left(\left(I_{0}\right)_{d B}\right) \leq \chi .
$$

Substituting (12) into (13) we get

$$
\left(P_{0}\right)_{d B} \leq\left(I_{0}\right)_{d B}+\frac{\alpha}{2} F_{(L)_{d B}}^{-1}(\chi)
$$

where $F_{(L)_{d B}}^{-1}(x)$ is the inverse function of $F_{(L)_{d B}}(x)$ given by (11). From (14) we can see that the maximum received signal 
power at the secondary BS is bounded by a function of the interference outage constraint parameters $I_{0}$ and $\chi$. Taking the maximum value of $\left(P_{0}\right)_{d B}$ given in (14), we can then evaluate the capacity of the secondary network as

$$
C=\log _{2}\left(1+10^{\frac{\left(P_{0}\right)_{d B}-(\Omega)_{d B}}{10}}\right)
$$

where $(\Omega)_{d B}=10 \log _{10} \Omega$ is the total interference and noise power perceived at the BS in the $\mathrm{dB}$ scale.

\section{CAPACITY UNDER REALISTIC CHANNEL MODELS}

In this section, we adopt more realistic channel models to include the effects of not only the pathloss but also shadowing and fading.

\section{A. Realistic Channel Models}

Modifying the simple channel models in (4) and (5), we can give the fading channel models as

$$
\begin{gathered}
h^{I}=K^{I} \xi^{I} \eta^{I} /\left(d^{I}\right)^{\alpha} \\
h^{A}=K^{A} \xi^{A} \eta^{A} /\left(d^{A}\right)^{\alpha}
\end{gathered}
$$

where $\xi^{I}$ and $\eta^{I}$ are random variables which model the effects of the shadowing and mutlipath fading in the interference channels, respectively. Similarly, $\xi^{A}$ and $\eta^{A}$ represent random shadowing and fading factors in the access channels, respectively. We assume that the shadowing factors $\xi^{I}$ and $\xi^{A}$ are mutually independent, each follows a log-normal distribution with a zero mean and a standard deviation $\sigma_{\xi}$ ranging from 5 to $12 \mathrm{~dB}$ with $8 \mathrm{~dB}$ being the typical value for macrocellular applications [16]. We further assume that the fading factors $\eta^{I}$ and $\eta^{A}$ are also mutually independent and follow identical distributions $f_{\eta}(x)$. When the channels are Nakagami faded, $f_{\eta}(x)$ is given by a Gamma distribution [16]

$$
f_{\eta}(x)=\frac{m^{m} x^{m-1}}{\Gamma(m)} \exp (-m x) \quad m \geq \frac{1}{2}
$$

where $m$ is the Nakagami shape factor and $\Gamma(\cdot)$ denotes the gamma function.

Let $\kappa^{I}=\xi^{I} \eta^{I}$ and $\kappa^{A}=\xi^{A} \eta^{A}$ denote the composite shadowing and fading factor of the interference channel and access channel, respectively. It follows that both $\kappa^{I}$ and $\kappa^{A}$ follow the same Gamma-log-normal distribution whose probability density function (PDF) is denoted as $f_{\kappa}(x)$ [16]. Such a Gamma-log-normal distribution can be approximated by a log-normal distribution as [16]

$$
f_{\kappa}(x) \approx \frac{1}{\epsilon \sqrt{2 \pi} \sigma x} \exp \left\{-\frac{\left(10 \log _{10} x-\mu\right)^{2}}{2 \sigma^{2}}\right\} .
$$

In (19), the mean $\mu$ and variance $\sigma^{2}$ are given by [16]

$$
\begin{gathered}
\mu=\epsilon^{-1}[\psi(m)-\ln (m)] \\
\sigma^{2}=\epsilon^{-2} \zeta(2, m)+\sigma_{\xi}^{2}
\end{gathered}
$$

respectively, where $\epsilon=\ln (10) / 10$ is a constant. In (20), $\psi(\cdot)$ is the Euler psi function given by

$$
\psi(m)=-X+\sum_{k=1}^{m-1} \frac{1}{k} \quad(m=1,2, \ldots)
$$

where $X \approx 0.5772$ is Euler's constant. In $(21), \zeta(\cdot, \cdot)$ is Riemann's zeta function and we have [16]

$$
\zeta(2, m)=\sum_{k=0}^{\infty} \frac{1}{(m+k)^{2}}(m=1,2, \ldots) .
$$

When $m=1$ this approximation is valid for $\sigma_{\xi}>6 \mathrm{~dB}$ and for $m>2$ the approximation is valid for all ranges of $\sigma_{\xi}$ of interest [16]. The effect of Nakagami fading is to decrease the mean $\mu$ and increase the variance $\sigma^{2}$. Such an approximation allows us to use a single formula (19) to represent both pure-shadowing and composite shadowing and fading channels. When both shadowing and fading are in concern, we use (19) with $\mu$ in (20) and $\sigma^{2}$ in (21). In case only the shadowing is of interest, we can still use (19) with $\mu=0$ and $\sigma=\sigma_{\xi}$. From (19), using the transformation of random variables we can show that both $\left(\kappa^{A}\right)_{d B}=10 \log _{10} \kappa^{A}$ and $\left(\kappa^{I}\right)_{d B}=10 \log _{10} \kappa^{I}$ have the same Gaussian PDF given by

$$
f_{(\kappa)_{d B}}(x) \approx \frac{1}{\sqrt{2 \pi} \sigma} \exp \left(-\frac{(x-\mu)^{2}}{2 \sigma^{2}}\right) .
$$

\section{B. Capacity}

Substituting (16) and (17) into (2) we have

$$
I=P_{0} \frac{K^{I}}{K^{A}}\left(\frac{d^{A}}{d^{I}}\right)^{\alpha} \frac{\kappa^{I}}{\kappa^{A}} .
$$

In the $\mathrm{dB}$ scale $(25)$ can be written as

$$
(I)_{d B}=\left(P_{0}\right)_{d B}-(L)_{d B}+\left(\kappa^{I}\right)_{d B}-\left(\kappa^{A}\right)_{d B}
$$

where $(I)_{d B},\left(P_{0}\right)_{d B}$, and $(L)_{d B}$ are given in (10), $\left(\kappa^{I}\right)_{d B}$ and $\left(\kappa^{A}\right)_{d B}$ are corresponding $\mathrm{dB}$ values with respect to $\kappa^{I}$ and $\kappa^{A}$. In (26), $(L)_{d B}$ is a random variable whose PDF $f_{(L)_{d B}}(x)$ can be obtained by differentiating its CDF $F_{(L)_{d B}}(x)$ given in (11). In addition, $\left(\kappa^{I}\right)_{d B}$ and $\left(\kappa^{A}\right)_{d B}$ are independent Gaussian random variables whose PDF is given by (24). Let us define $(T)_{d B}=-(L)_{d B}+\left(\kappa^{I}\right)_{d B}-\left(\kappa^{A}\right)_{d B}$. Its PDF $f_{(T)_{d B}}(x)$ can be obtained as the convolution of the individual PDFs of $(L)_{d B},\left(\kappa^{I}\right)_{d B}$, and $\left(\kappa^{A}\right)_{d B}$. From $f_{(T)_{d B}}(x)$, we can get the corresponding $\mathrm{CDF} F_{(T)_{d B}}(x)$ through numerical integration. Since $\left(I_{0}\right)_{d B}=(P)_{d B}+(T)_{d B}$, it follows that the CDF of $\left(I_{0}\right)_{d B}$ is related to $F_{(T)_{d B}}(x)$ by

$$
F_{(I)_{d B}}=F_{(T)_{d B}}\left(x-\left(P_{0}\right)_{d B}\right) .
$$

Considering the interference outage constraint specified in (13), from (27) it is easy to show that $\left(P_{0}\right)_{d B}$ is bounded by

$$
\left(P_{0}\right)_{d B} \leq\left(I_{0}\right)_{d B}-F_{(T)_{d B}}^{-1}(1-\chi)
$$

where $F_{(T)_{d B}}^{-1}(\cdot)$ is the inverse function of $F_{(T)_{d B}}(\cdot)$. Finally, taking the maximum value of $\left(P_{0}\right)_{d B}$ given by (28), we can evaluate the uplink cell capacity according to (15). 


\section{NUMERICAL RESULTS AND DISCUSSIONS}

Numerical results will be presented in this section to visualize the relationship between the cell capacity $C$ and the interference outage constraint defined on two parameters: the power threshold (in $\mathrm{dB})\left(I_{0}\right)_{d B}$ and outage limit $\chi$. As revealed by (14) and (28), the impact of $\left(I_{0}\right)_{d B}$ on the capacity $C$ is relatively straightforward since $\left(I_{0}\right)_{d B}$ is additive to $\left(P_{0}\right)_{d B}$. In contrast, the impact of $\chi$ on $C$ is more obscure and is thus our focus in this section. In the following numerical studies, we assume $\left(I_{0}\right)_{d B}=0 \mathrm{~dB}$ and compute the capacity $C$ as a function of $\chi$.

Based on (14) and (15), Fig. 2 shows the capacity $C$ as a function of $\chi$ with simple pathloss-only channel models. As expected, the capacity increases with increasing outage limit $\chi$. A bigger value of $\chi$ means that the primary network is more tolerant to the interference, thereby allowing the secondary network to gain more capacity. Moreover, since the interference outage constraint is imposed on the "sampled" interfere perceived at an arbitrary primary receiver, the capacity is shown to be related to the exact location of the primary receiver in concern. Fig. 2 shows that with small values of $\chi$, the capacity is limited by primary receivers near the cell edge (larger values of $r / R$ ). In contrast, when $\chi$ grows larger, the capacity becomes limited by primary receivers close to the cell center (smaller values of $r / R$ ). When there are multiple primary users in the cell, the interference outage constraint should correspondingly be enforced in multiple locations. In this case, the capacity should be chosen as the minimum among all the capacities computed under multiple interference outage constraints.

Changing to realistic channel models, Fig. 3 shows the capacity $C$ as a function of $\chi$ based on (15) and (28). We first consider a channel model with pathloss and shadowing regardless of small scale fading. The shadowing standard deviation $\sigma_{\xi}$ is set to be $8 \mathrm{~dB}$. Comparing Fig. 3 with Fig. 2, we found that log-normal shadowing tends to reduce the capacity when $\chi$ is small, but increases the capacity when $\chi$ becomes larger. Similar to Fig. 2, the capacity is shown to be related to the location of the primary receiver in concern. However, unlike Fig. 2, the capacity seems to be constantly limited by primary users near the cell center. This suggests that in order to maximize the capacity of such a secondary network, the secondary BS should be located as further away to any primary receivers as possible.

Finally, the impact of small scale fading on the capacity is shown in Fig. 4 . We set $\sigma_{\xi}=8 \mathrm{~dB}, k=0.7$, and vary the value of the Nakagami shape factor $m$ to represent different fading scenarios. The case of $m=1$ corresponds to Rayleigh fading, whereas $m=10000$ approximates a shadowing channel where there is no small scale fading. Similar to that of log-normal shadowing, it is observed that fading has an impact to reduce the capacity when $\chi$ is small and increase the capacity when $\chi$ becomes larger. However, such an impact is trivial as we observe that the capacities obtained with $m=1$ have little differences from those obtained with $m=10000$.

\section{CONCLUSIONS}

In this paper, we have studied the capacity of a secondary network with a constraint on the outage of the interference power perceived by an arbitrary primary receiver. We have considered a secondary network where uniformly distributed secondary users communicate with a central BS using the TDMA scheme. A power control scheme is assumed which maintains the received signal power at the $\mathrm{BS}$ as $P_{0}$. We have studied the interference raised by such a secondary network and characterised its distribution. Based on this, we have further studied the capacity of the secondary network under an interference outage constraint specified by a threshold $I_{0}$ and an outage probability $\chi$. The results have shown that with small values of $\chi$, the capacity is limited by primary users near the cell edge. In contrast, when $\chi$ grows larger, the capacity becomes limited by primary users near the cell center. It has been found that shadowing and fading result in similar impacts on reducing the capacity when $\chi$ is small while increasing the capacity when $\chi$ becomes larger. However, compared with shadowing, fading only has trivial impacts on the capacity .

\section{APPENDIX \\ DERIVATION OF (7)}

In this Appendix, we wish to find the CDF $F_{L}(x)$ of the random variable $L$ given by

$$
L=\left(d^{I} / d^{A}\right)^{2}
$$

where $d^{A}$ and $d^{I}$ are distances from the target secondary user to the secondary BS and the primary user, respectively. As illustrated in Fig. 1, secondary users are uniformly distributed in a circular secondary cell of radius $R$. The BS is at the center of the cell and the distance between the primary user and the secondary BS is $r$.

One approach to find the PDF of $L$ is by using transformations of random variables. This approach, however, leads to a definite integration which cannot be solved in a closed-form. Instead, a geometric-based method will be introduced in this Appendix to give a closed-form expression of $F_{L}(x)$. First of all, as shown in Fig. 5, we put the secondary network into a $(x, y)$ coordinate system. The coordinates of the BS (point $O_{1}$ ), primary user (point $P$ ) and secondary user are given by $(r / 2,0),(-r / 2,0)$, and $(x, y)$, respectively. We consider this coordinate system as a probability plane corresponding to the location distribution of the secondary users. Since the secondary user are uniformly distributed within the circle $O_{1}$, the disk area $O_{1}$ has a probability density of $1 / R^{2}$ and elsewhere the probability density is zero.

Under this coordinate system, (29) can be rewritten as

$$
L=\frac{(x+r / 2)^{2}+y^{2}}{(x-r / 2)^{2}+y^{2}}
$$

The set of the points $(x, y)$ that fulfill (30) forms a circle $\mathrm{O}_{2}$ with an origin located at $\left(-\frac{r}{2}\left(\frac{1+L}{1-L}\right), 0\right)$ and a radius

$$
\tilde{R}=\frac{\sqrt{L} r}{|1-L|} .
$$


The CDF of $L$ can be obtained by investigating the relationships between circle $O_{1}$ and circle $O_{2}$. For convenience, let us denote $k=r / R$. With $L$ increase from 0 to $\infty$, we have the following five stages:

1) When $L$ increases from 0 to $(1-k)^{2}$, circle $O_{2}$ starts as a small circle around point $P$ and become a circle that internally tangents circle $O_{1}$. The CDF $F_{L}(x)$ is area of disk $\mathrm{O}_{2}$ times the probability density, i.e.,

$$
F_{L}(x)=\frac{\tilde{R}^{2}}{R^{2}}, \quad x \in\left[0,(1-k)^{2}\right]
$$

2) When $L$ increases from $(1-k)^{2}$ to 1 , circle $O_{2}$ intersects circle $O_{1}$. The CDF $F_{L}(x)$ is the common area of circles $O_{1}$ and $O_{2}$, denoted as $S$, times the probability density, i.e.,

$$
F_{L}(x)=\frac{S}{\pi R^{2}}, \quad x \in\left[(1-k)^{2}, 1\right)
$$

where $S$ can be easily obtained using basic geometrics

$$
S=\theta_{1} R^{2}+\theta_{2} \tilde{R}^{2}-D R \sin \left(\theta_{1}\right) .
$$

In (34), we have

$$
\begin{aligned}
& \theta_{1}=\arccos \left(\frac{R^{2}+D^{2}-\tilde{R}^{2}}{2 R D}\right) \\
& \theta_{2}=\arccos \left(\frac{\tilde{R}^{2}+D^{2}-R^{2}}{2 \tilde{R} D}\right),
\end{aligned}
$$

and

$$
D=\frac{r}{|1-x|}
$$

is the distance between point $O_{1}$ and point $O_{2}$.

3) When $L=1$, the $\operatorname{CDF} F_{L}(x)$ is the probability density times the area which is within circle $O_{1}$ and left to the $\mathrm{y}$-axis. Using basic geometrics we can get

$$
F_{L}(x)=\frac{1}{\pi} \arccos \left(\frac{r}{2 R}\right), \quad x=1
$$

4) When $L$ increases from 1 to $(1+k)^{2}$, circle $O_{2}$ intersects circle $O_{1}$. The complementary CDF $1-F_{L}(x)$ is the common area $S$ times the probability density. It follows that the CDF is given by

$$
F_{L}(x)=1-S /\left(\pi R^{2}\right), \quad x \in\left(1,(1+k)^{2}\right)
$$

where $S$ is given by (34).

5) When $L$ increases from $(1+k)^{2}$ to $\infty$, circle $O_{2}$ is inside circle $O_{1}$ and gradually converges toward point $\mathrm{O}_{2}$. The complementary CDF $1-F_{L}(x)$ is given by the area of disk $\mathrm{O}_{2}$ times the probability density. It follows that the $\mathrm{CDF}$ is given by

$$
F_{L}(x)=1-\frac{\tilde{R}^{2}}{R^{2}}, \quad x \in\left((1+k)^{2}, \infty\right) .
$$

Finally, (7) is obtained by substituting (31) and (34) into (32), (33), (38), (39), and (40).

\section{REFERENCES}

[1] Q. Zhao and B. M. Sadler, "A survey of dynamic spectrum access," IEEE Signal Processing Mag., vol. 24, no. 3, pp. 79-89, May 2007.

[2] H. H. Chen, and M. Guizani, Next Generation Wireless Systems and Networks, Chichester: John Wiley \& Sons, 2006.

[3] S. Haykin, "Cognitive radio: brain-empowered wireless communications", IEEE J. Selected Areas Commun., vol. 23, no. 2, pp. 201-220, Feb. 2005.

[4] I. F. Akyildiz, W. Y. Lee, M. C. Vuran and S. Mohanty, "NeXt generation/dynamic spectrum access/cognitive radio wireless networks: a survey", Computer Networks, vol. 50, no. 13, pp. 2127-2159, Sept. 2006.

[5] S. Srinivasa and S. A. Jafar, "The throughput potential of cognitive radio: a theoretical perspective," IEEE Commun. Mag., vol. 45, no. 5, pp. 73-79, May 2007.

[6] Federal Communication Commission, "Facilitating opportunities for flexible, efficient, and reliable spectrum use employing cognitive radio technologies", NPRM \& Order, ET Docket No. 03-108, FCC 03-322, 30 Dec. 2003.

[7] T. Clancy. "Formalizing the interference temperature model," Wiley J. Wireless Commun. and Mobile Computing, vol. 7, no. 9, pp. 1077-1086, Nov. 2007.

[8] J. Bater, H.-P. Tan, K. N. Brown, L. Doyle, "Modelling interference temperature constraints for spectrum access in cognitive radio networks," Proc. IEEE ICC'07, Glasgow, UK, June 2007, pp. 6493-6498.

[9] S. A. Jafar, and S. Srinivasa, "Capacity limits of cognitive radio with distributed and dynamic spectral activity," Proc. IEEE ICC'06, Istanbul, Turkey, June 2006, pp. 5742-5747.

[10] X. Hong, C.-X. Wang, and J. Thompson, "Interference modelling of cognitive radio networks in spectrum sharing systems", Proc. IEEE VTC'08-Spring, Singapore, 11-14 May 2008, accepted.

[11] M. Gastpar, "On capacity under receive and spatial spectrum-sharing constraints", IEEE Trans. Inform. Theory, vol. 53, no. 2, pp. 471-487, Feb. 2007.

[12] A. Ghasemi, and E. S. Sousa, "Capacity of fading channels under spectrum-sharing constraints", Proc. IEEE ICC'06, Istanbul, Turkey, June 2006, pp. 4373-4378.

[13] X. Hong, C.-X. Wang, and J. Thompson, "Uplink cell capacity of cognitive radio networks with peak interference power constraints", Proc. IEEE ICCCAS'08, Xiamen, China, May 2008, submitted.

[14] C.-X. Wang, X. Hong, H.-H. Chen, and J. S. Thompson, "On capacity of cognitive radio networks under average interference power constraints", IEEE Trans. Wireless Commun., submitted.

[15] X. Hong, C.-X. Wang, H.-H. Chen, and J. Thompson, "On capacity of cognitive radio networks with average interference power constraints", Proc. IEEE ICC'2008, Beijing, China, May 2008, accepted.

[16] G. L. Stuber, Principles of Mobile Communication, 2nd Edition, Boston: Kluwer Academic Publishers, 2001.

[17] S. Mangold, A. Jarosch, C. Monney, "Operator assisted cognitive radio and dynamic spectrum assignment with dual beacons - detailed evaluation," Proc. First Int. Conf. on Commun. Systems Software and Middleware, Delhi, India, Jan. 2006, pp. 1-6.

[18] B. Wild and K. Ramchandran, "Detecting primary receivers for cognitive radio applications", Proc. IEEE DySPAN'05, Baltimore, USA, Nov. 2005, pp. 124-130.

[19] C.-X. Wang, H.-H. Chen, X. Hong, and M. Guizani, "Policy-based management for cognitive radio networks", IEEE Veh. Techno. Mag., accepted.

[20] A. Papoulis and S. U. Pillai, Probability, Random Variables and Stochastic Processes, 4th ed. New York: McGraw-Hill, 2002. 


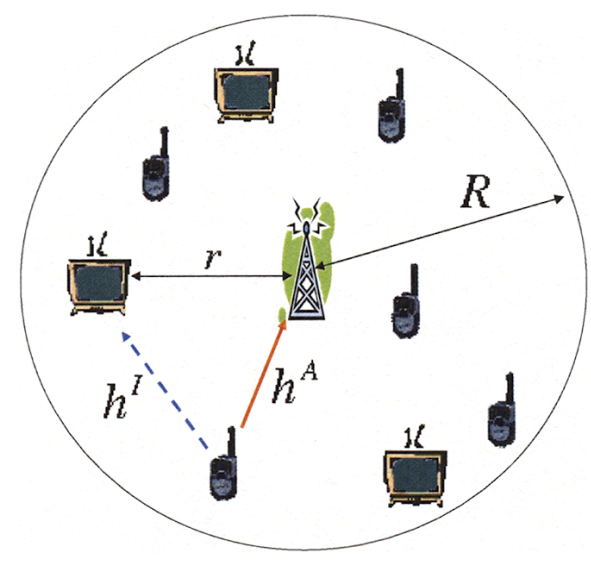

Fig. 1. System model.

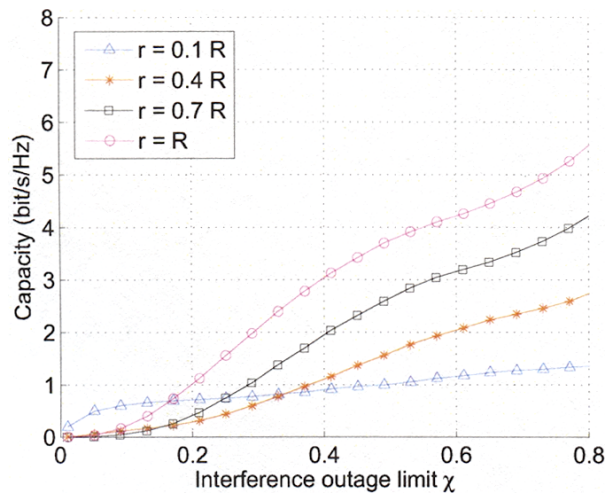

Fig. 2. Capacity as a function of $\chi$ with different values of $r / R$ $\left(\left(I_{0}\right)_{d B}=0 \mathrm{~dB}\right.$, pathloss-only channel model).

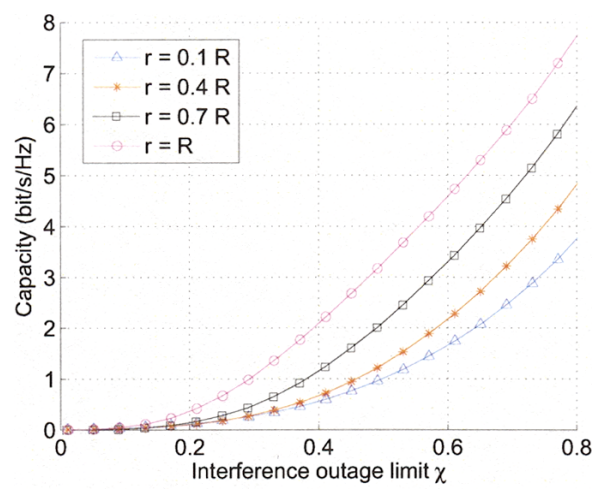

Fig. 3. Capacity as a function of $\chi$ with different values of $r / R$ $\left(\left(I_{0}\right)_{d B}=0 \mathrm{~dB}, \sigma_{\xi}=8 \mathrm{~dB}\right.$, pathloss-shadowing channel model $)$.

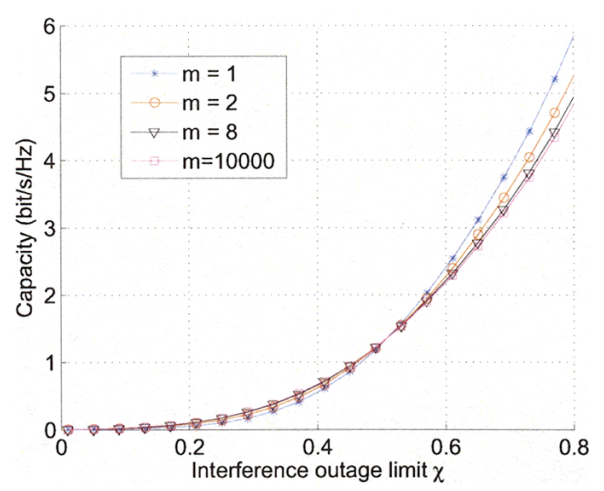

Fig. 4. Capacity as a function of $\chi$ with different values of $m$ $\left(\left(I_{0}\right)_{d B}=0 \mathrm{~dB}, \sigma_{\xi}=8 \mathrm{~dB}, r / R=0.7\right.$, pathloss-shadowing-fading channel model).

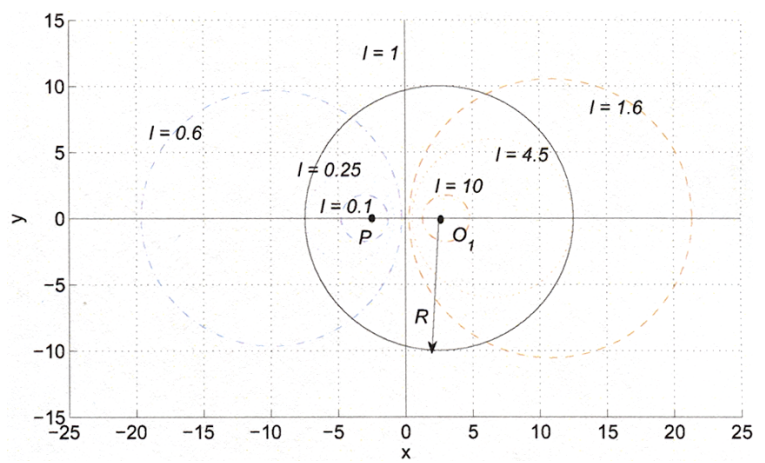

Fig. 5. Illustration of the geometric-based approach in the Appendix. 